\title{
Multi-contrast photoacoustic microscopy
}

S. Hu, R. Sohn, Z.-H. Lu, B. Soetikno, Q. Zhong, et al.

S. Hu, R. Sohn, Z.-H. Lu, B. Soetikno, Q. Zhong, J. Yao, K. Maslov, J. M. Arbeit, L. V. Wang, "Multi-contrast photoacoustic microscopy," Proc. SPIE 8223, Photons Plus Ultrasound: Imaging and Sensing 2012, 822310 (15 March 2012); doi: 10.1117/12.909754

SPIE. Event: SPIE BiOS, 2012, San Francisco, California, United States 


\title{
Multi-contrast Photoacoustic Microscopy
}

\author{
S. Hu ${ }^{1}$, R. Sohn ${ }^{2}$, Z.-H. Lu ${ }^{2}$, B. Soetikno ${ }^{1}$, Q. Zhong ${ }^{1}$, J. Yao ${ }^{1}$, K. Maslov ${ }^{1}$, \\ J. M. Arbeit ${ }^{2, *}$, and L. V. Wang ${ }^{1, *}$ \\ ${ }^{1}$ Department of Biomedical Engineering, Washington University in St. Louis, St. Louis, MO 63130, USA \\ ${ }^{2}$ Siteman Cancer Center, Washington University School of Medicine, St. Louis, MO, USA \\ ${ }^{*}$ Correspondence should be addressed to JMA (tumor biology; arbeitj@wudosis.wustl.edu) and LVW \\ (photoacoustics; lhwang@biomed.wustl.edu).
}

\begin{abstract}
We developed multi-contrast photoacoustic microscopy (PAM) for in vivo anatomical, functional, metabolic, and molecular imaging. This technical innovation enables comprehensive understanding of the tumor microenvironment. With multi-contrast PAM, we longitudinally determined tumor vascular anatomy, blood flow, oxygen saturation of hemoglobin, and oxygen extraction fraction.
\end{abstract}

Keywords: Optical-resolution photoacoustic microscopy, vascular anatomy, hemoglobin oxygen saturation, oxygen extraction fraction, tumor microenvironment.

\section{INTRODUCTION}

To date, photoacoustic imaging of anatomical (1), functional (2), metabolic (3), molecular (4), and genetic (5) contrasts has been demonstrated in vivo. However, each individual contrast can hardly provide a comprehensive characterization of the complex biological system. In cancer research, for instance, vascular anatomy, blood oxygenation, and the metabolic rate of oxygen are highly desired to study neovascularization, tumor hypoxia, and tumor hypermetabolism, respectively. In some disease models, exogenous molecular biomarkers are needed to visualize non-absorbing molecules (6). By integrating the aforementioned multiple photoacoustic contrasts into a single microscopy platform, we have developed multi-contrast photoacoustic microscopy (PAM). Its potential application includes vascular and metabolic signaling pathways, neurovascular and neurometabolic coupling, in vivo drug screening, as well as therapy design and management.

\section{METHODS AND MATERIALS}

The multi-contrast imaging platform was developed based on our second-generation optical-resolution PAM (Fig. 1) (7). The object to be imaged is irradiated with a short-pulsed laser beam generated by a wavelength-tunable laser system, consisting of an Nd:YLF pump laser and a dye laser. Wideband ultrasonic waves are induced as a result of transient thermo-elastic expansion due to the laser excitation, and then detected by a high-frequency ultrasonic transducer. To maximize the sensitivity, the optical illumination and the ultrasonic detection in our system are configured confocally by an acoustic-optical beam combiner, where a right-angle prism and a rhomboid prism form a cube with a thin layer of silicone oil in between. This novel design offers high acoustic detection sensitivity. A microscopic objective lens is employed to achieve nearly diffraction-limited optical resolution $(\sim 2.6 \mu \mathrm{m}$ in clear media).

The wavelength-tunable laser allows exciting various endogenous chromophores (Fig. 2A), and exogenous molecular biomarkers (Fig. 2C). The diffraction-limited optical focusing provides a cellular resolution. The two-dimensional actuator-based mechanical scanning and steering-mirror-based optical scanning enable wide-field and real-time imaging, respectively. Multi-wavelength measurement allows quantifying hemoglobin oxygen saturation $\left(\mathrm{sO}_{2}\right)(\mathrm{Fig}$. 2B) $(8)$. The photoacoustic Doppler effect reveals blood flow (9). Based on the anatomical and functional information, oxygen

Photons Plus Ultrasound: Imaging and Sensing 2012, edited by Alexander A. Oraevsky, Lihong V. Wang, Proc. of SPIE Vol. 8223, 822310 - (C) 2012 SPIE · CCC code: 1605-7422/12/\$18 · doi: 10.1117/12.909754 
extraction fraction (OEF) and the metabolic rate of oxygen $\left(\mathrm{MRO}_{2}\right)$ can be computed (3). Further, powerful vessel segmentation analysis allows us to assess the multiple physiological contrasts on a single-vessel basis $(10,11)$.

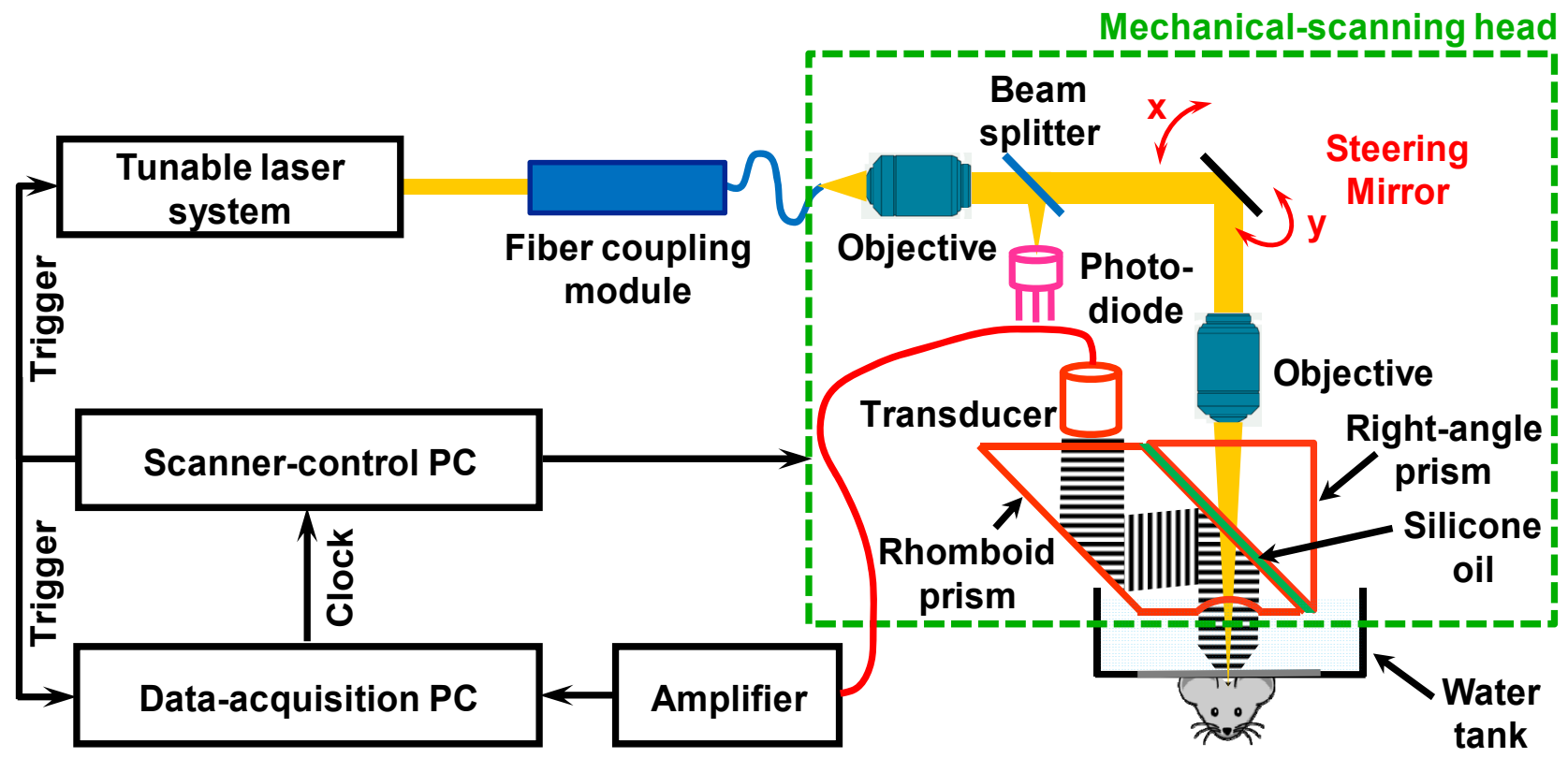

Fig. 1. Schematic of the multi-contrast PAM system.
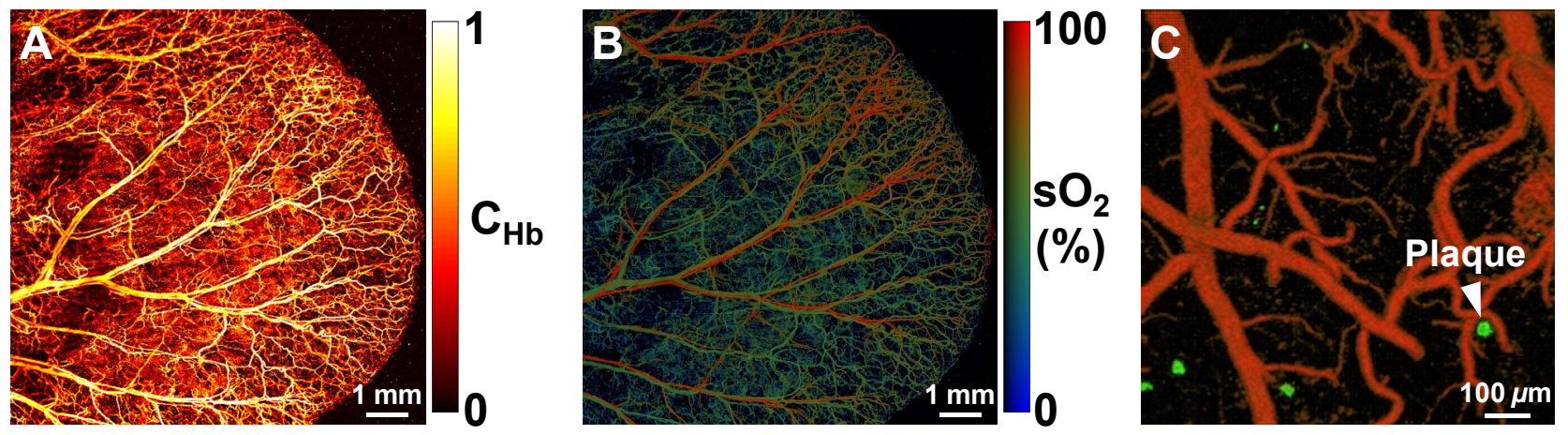

Fig. 2. Multi-contrast PAM in vivo. The vascular anatomy and function in a mouse ear are shown by the maps of (A) hemoglobin concentration $\left(\mathrm{C}_{\mathrm{Hb}}\right)$ and $(\mathrm{B}) \mathrm{sO}_{2}$, respectively. (C) Molecular imaging of Congo Red dye-labeled cerebral amyloid plaques in an Alzheimer's disease mouse model.

\section{RESULTS}

Recently, we have applied multi-contrast PAM to longitudinally monitor the regulation of angiogenesis and the tumor metabolic microenvironment during spontaneous growth and chemotherapy in a renal carcinoma xenograft (Fig. 3). Right after taking the baseline measurements of the vascular anatomy and $\mathrm{sO}_{2}$, renal carcinoma cells were inoculated into the mouse ear. Then, the spontaneous tumor growth was monitored for 25 days before a daily RAD001 treatment for two weeks. During the tumor growth and treatment, vascular anatomical (i.e., diameter) and functional (i.e., blood flow and $\mathrm{sO}_{2}$ ) information was acquired, based on which $\mathrm{OEF}$ was computed. A marked normalization of arterial blood flow in response to the 2-week RAD001 treatment was clearly observed, which also implied the normalization of tumor oxygen metabolism. 

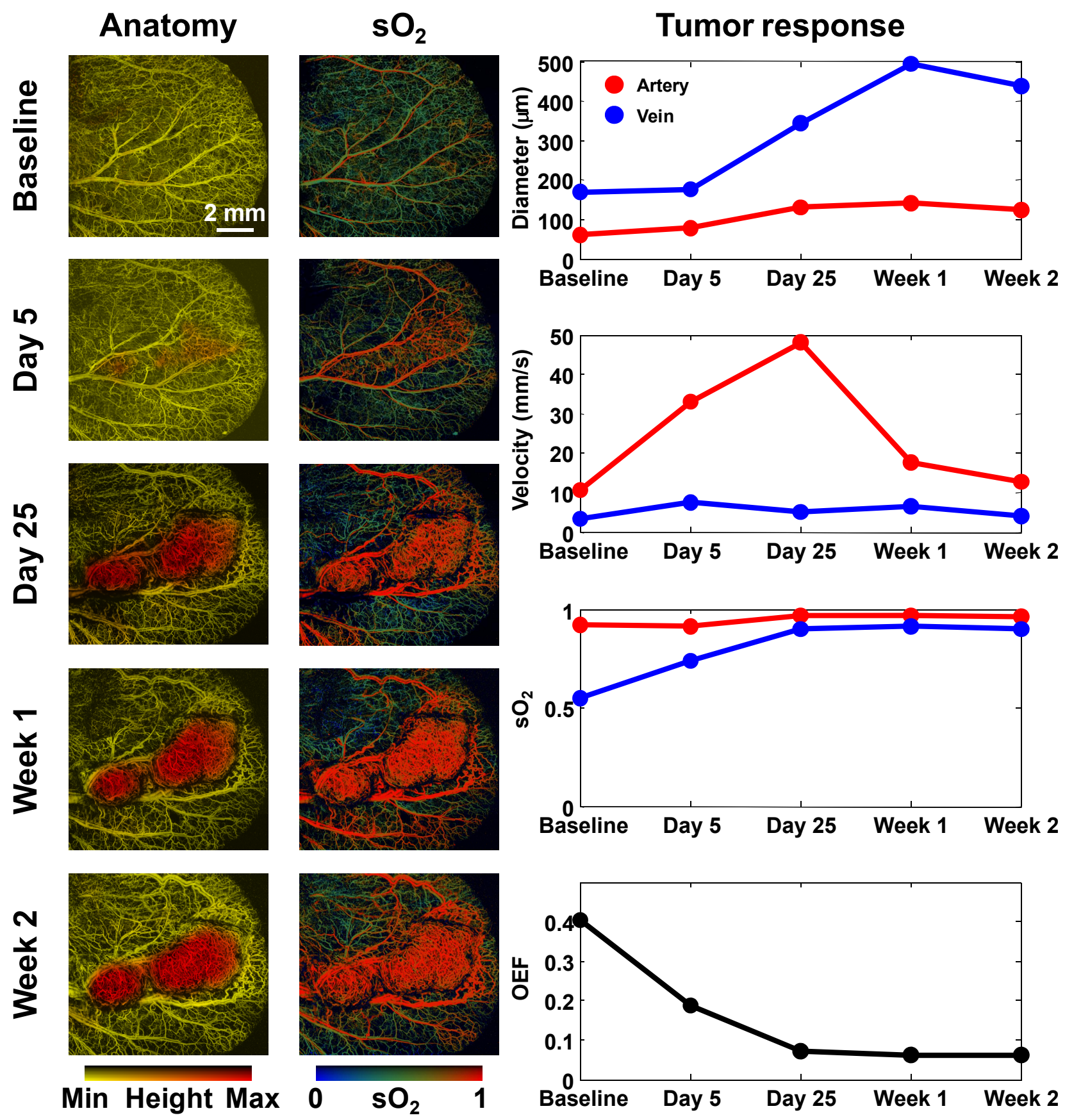

Fig. 3. Multi-contrast PAM of the tumor microenvironment during spontaneous growth and chemotherapy. 


\section{CONCLUSION}

We have developed multi-contrast PAM for in vivo imaging of anatomical, functional, metabolic, and molecular contrasts at the microscopic level. Its potential has been demonstrated by our recent research on the microenvironmental regulation of renal tumor during spontaneous growth and chemotherapy. Future applications of multi-contrast PAM include vascular and metabolic signaling pathways, neurovascular and neurometabolic coupling, in vivo drug screening, as well as therapy design and management.

\section{ACKNOWLEDGMENTS}

This work was sponsored by National Institutes of Health Grants R01 EB000712, R01 EB008085, R01 CA134539, U54 CA136398, R01 CA157277, R01 EB010049, and R01 CA159959. L.V.W. has financial interests in Microphotoacoustics, Inc. and Endra, Inc., which, however, did not support this work.

\section{REFERENCES}

[1] H. P. Brecht et al., J. Biomed. Opt. 14, 064007 (2009).

[2] H. F. Zhang, K. Maslov, M. Sivaramakrishnan, G. Stoica, L. V. Wang, Appl. Phys. Lett. 90, 3 (2007).

[3] J. Yao, K. I. Maslov, Y. Zhang, Y. Xia, L. V. Wang, J. Biomed. Opt. 16, 076003 (2011).

[4] V. Ntziachristos, D. Razansky, Chem. Rev. 110, 2783 (2010).

[5] L. Li, H. F. Zhang, R. J. Zemp, K. Maslov, L. V. Wang, J. Innov. Opt. Health Sci. 1, 207 (2008).

[6] S. Hu, P. Yan, K. Maslov, J.-M. Lee, L. V. Wang, Opt. Lett. 34, 3899 (2009).

[7] S. Hu, K. Maslov, L. V. Wang, Opt. Lett. 36, 1134 (2011).

[8] S. Hu, K. Maslov, L. V. Wang, J. Vis. Exp., (2011).

[9] J. Yao, K. Maslov, Y. Shi, L. A. Taber, L. V. Wang, Opt. Lett. 35, 1419 (2010).

[10] S. Oladipupo et al., Proc. Natl. Acad. Sci. USA 108, 13264 (2011).

[11] S. S. Oladipupo et al., Blood 117, 4142 (2011). 